\title{
Upregulation of microRNA-450 inhibits the progression of lung cancer in vitro and in vivo by targeting interferon regulatory factor 2
}

\author{
FABING LIU, XIAOBO YU, HAIHUA HUANG, XI CHEN, JIN WANG, XIAOMIAO ZHANG and QIANG LIN
}

Department of Thoracic Surgery, Shanghai General Hospital, Shanghai Jiaotong University

School of Medicine, Shanghai 200080, P.R. China

Received October 29, 2015; Accepted May 16, 2016

DOI: $10.3892 /$ ijmm.2016.2612

\begin{abstract}
MicroRNAs (miRNAs) are a class of non-coding RNAs that play pivotal roles in human lung cancer development. The majority of studies have focused on either non-small cell lung cancer (NSCLC) or small cell lung cancer (SCLC). In the present study, we investigated a plausible mechanism of action of miR-450 in these types of lung cancer. We found that the level of miR-450 was decreased in lung cancer cell lines, as well as in solid tumors. As exemplified in the H510A (SCLC) and H2291 (NSCLC) cells, transfection with lentivirus carrying miR-450 upregulated miR-450 expression and significantly attenuated lung cancer cell proliferation and invasion, as well as the growth of implantated tumors. Interferon regulatory factor 2 (IRF2) was also verified to be a direct target of miR-450 in lung cancer cells. The overexpression of IRF2 in the H510A and H2291 cells abrogated the inhibitory effects of miR-450 on lung cancer cell proliferation and invasion. Taken together, in this study, we identified a novel role of miR-450 in lung cancer. miR-450 targets IRF2 and thus supresses lung cancer cell proliferation and invasion.
\end{abstract}

\section{Introduction}

Lung cancer has been reported to be among the most deadly cancers worldwide (1). Non-small-cell lung cancer (NSCLC) accounts for most primary lung cancer (2). Instead, the smallcell lung cancer (SCLC) is a distinct form of lung cancer with unique clinical and histological characteristics, representing $\sim 15 \%$ of all new cases of lung cancer (2). The reported prognosis and five-year survival rates are terribly poor (1). In China, it has been shown that approximately over half a million people died from lung cancer each year (3).

Correspondence to: Dr Qiang Lin, Department of Thoracic Surgery, Shanghai General Hospital, Shanghai Jiaotong University School of Medicine, 100 Haining Road, Shanghai 200080, P.R. China

E-mail: linqiang_sjtu@163.com

Key words: lung cancer, microRNA-450, interferon regulatory factor 2, cancer proliferation, invasion
The microRNAs (miRNAs) denote myriads of noncoding RNAs with short length which suppress gene expression by base paring to the 3'-untranslated region (3'-UTR) of targets $(4,5)$. The miRNAs can either exist individually or as a cluster in the whole genome. Meanwhile, they can also reside in introns or exons of functional genes (6). To date, more than one thousand miRNAs have been defined. They can regulate multiple pathways associated with cancer by binding numerous target mRNAs (7). Although the detailed clues for the exact molecular mechanisms underlying lung cancer cell proliferation or metastasis are still elusive, recent researches have implied that miRNAs may play pivotal roles in lung cancer progression $(6,8,9)$. For instance, miR-451 was shown to be lowered in NSCLC tissues while raised levels of miR-451 are capable of suppressing NSCLC proliferation by inhibiting ras-related protein 14 (RAB14) and triggering apoptosis (10). miR-31 was found to be an oncogenic miRNA through promoting NSCLC growth and repressing tumor suppressor genes such as large tumor suppressor 2 (LATS2) and PP2A regulatory subunit $\mathrm{B} \alpha$ isoform (PPP2R2A) (11). Instead, miR-25 was reported to regulate SCLC progression by targeting CDK2 (12). The $\mathrm{miR}-34 \mathrm{~b} / \mathrm{c}$ has also been suggested to play an oncogenic role in SCLC (13). However, whether a single microRNA can play a role in both NSCLC and SCLC is largely elusive.

The interferon regulator factor 2 (IRF2) is a member of the interferon regulatory family and participates in various pathways related to tumorigenesis via modulating target gene expression (14). IRF2 was also reported to be the functional antagonist for IRF1 and regulate those genes with interferon regulatory element binding sites either as positive or negative factors (15). IRF2 has recently become an active area of research owing to its role in tumor progression $(16,17)$. For example, IRF2 can inhibit p21 and promote proliferation of cells as shown in leukemogenesis (18). In some type of cancer cells, aberrant expression of IRF2 was correlated with tumor growth, invasion and stage (19). Meanwhile, reducing IRF2 can decrease the expression of cyclin D1 while elevating IRF2 expression can enhance the malignancy of ESCC cells $(19,20)$. Some studies have implicated the role of IRF2 in tumor progression such as in pancreatic cancer and breast cancer although the exact molecular mechanisms remain to be clarified (21). 
In this study, we first showed that downregulating miR-450 in both NSCLC and SCLC cells and human tumors. We then explored the functional roles of miR-450 in shaping malignant phenotypes of NSCLC such as proliferation and invasion. Furthermore, we investigated the targeting effect of miR-450 on IRF2 and direct regulatory roles of IRF2 in miR-450 induced NSCLC and SCLC growth inhibition. Our present study may extend current understanding on the epigenetic regulation in human lung cancer.

\section{Materials and methods}

Lung cancer cells and human samples. There were 7 lung cancer cell lines used in this study, H1703, SPC-A1, H510A, H1299, H920, H522 and H2291. The SPC-A1 cell line was purchased from the Shanghai Institute of Cell Biology (Shanghai, China). The other cell lines were all commercially available from the American Type Culture Collection (ATCC, Shanghai, China). All the cell lines were NSCLC, apart from H510A, which was SCLC. A control cell line, normal human bronchial epithelial cell line (NuLi-1) was obtained from ATCC. All the cells were cultured in RPMI-1640 medium supplemented with $10 \%$ fetal bovine serum and $150 \mathrm{U} / \mathrm{ml}$ penicillin plus $150 \mu \mathrm{g} / \mathrm{ml}$ streptomycin (all from Sigma, Shanghai, China) in a culture chamber with $5 \% \mathrm{CO}_{2}$ at $37^{\circ} \mathrm{C}$. Human lung cancer samples were surgically retrieved from patients registered at Shanghai General Hospital between October 2014 and April 2015. The tumor (T) tissues and corresponding adjacent non-tumor (ANT) tissues were all paired samples. All patients provided written informed consent for the use of their samples. All surgical and experimental procedures related with human subjects were approved by the Human Research and Ethics Committee of Shanghai General Hospital, Shanghai Jiaotong University School of Medicine.

Reverse transcription-quantitative RT-PCR (RT-qPCR). To evaluate the expression of miR-450 and IRF2 in lung cancer, RT-qPCR was carried out. In brief, total RNA was extracted from both the NSCLC and SCLC cell lines, and human samples using TRIzol reagent (Sigma), and reverse transcribed into cDNA using a SYBR Premix Ex Taq ${ }^{\mathrm{TM}}$ kit (Takara Bio, Inc., Shiga, Japan) following the manufacturer's instructions. To detect the gene for miR-450, a TaqMan miRNA qRT-PCR kit (Applied Biosystems, Foster City, CA, USA) was applied. For quantifying IRF2 mRNA, a SYBR-Green PCR Master mix kit (Applied Biosystems) was used. In both cases, GAPDH was used as an internal control. All reactions were performed using an ABI PRISM ${ }^{\circledR} 7000$ Sequence Detection system (Applied Biosystems) following the manufacturer's instructions. Relative expression levels were shown which denote the fold change relative to the levels under control conditions.

MicroRNA-450 transfection. We used a lentiviral transfection system to ectopically upregulate miR-450 in the SCLC and NSCLC cell lines (H510A and H2291 cells, respectively). The lentivirus covering human miR-450 mimics (Lenti-miR-450), or a negative control miRNA (Lenti-C) were purchased from Sigma. The sequences were as follows: miR-450 sense, 5'-TTTTTGCGATGTGTTCCTAATG-3' and antisense, 5'-GATATGCCACGGGTTAGATT-3'; Lenti-C sense, 5'-CTC GCTTCTCGAGCACA-3' and antisense, 5'-AACGCTTCA
CGAAGGTTCGT-3'. The transduction of lentivirus into the H510A and H2291 were performed using Lipofectamine 2000 reagent (Invitrogen Life Technologies, Carlsbad, CA, USA) according to the manufacturer's instructions. After $24 \mathrm{~h}$, the cultured medium was removed and RT-qPCR was used to confirm the efficiency of transfection.

Cell proliferation assay. A 3-(4,5-dimethylthiazol-2-yl)-2,5-diphenyltetrazolium bromide (MTT) assay (Sigma) was utilized to examine cell proliferation. In brief, $24 \mathrm{~h}$ following transfection, the H510A and H2291 cells were all suspended and plated in 96 -well plates $\left(10^{4}\right.$ cells/well) for 5 days. Subsequently, $20 \mu 1$ MTT solution $(5 \mathrm{mg} / \mathrm{ml})$ were added to the culture every $24 \mathrm{~h}$ and maintained for $4 \mathrm{~h}$. Crystalline formazan was dissolved in $150 \mu \mathrm{l}$ sodium dodecyl sulfate sodium salt (SDS, 15\%) solution for $24 \mathrm{~h}$. The optical density (OD) at $490 \mathrm{~nm}$ was evaluated using a Spectramax M5 microplate reader (Molecular Devices, Sunnyvale, CA, USA) following the instructions provided by the manufacturer.

Cell invasion assays. Chemotaxis 96-well Transwell assay (Qiangen, Inc., Valencia, CA, USA) was utilized to evaluate cell invasion. The upper chamber was first coated with Matrigel (Invitrogen, Shanghai, China) overnight. The lentivirus-transfected H510A or H2291 cells were suspended and plated into the upper chamber $\left(10^{4}\right.$ cells/well $)$ in RPMI-1640 medium (Sigma) without serum. The lower chambers were filled with RPMI-1640 medium plus additional $2 \%$ fetal bovine serum (FBS). After $24 \mathrm{~h}$ of incubation, all upper chambers were replenished and the cells migrating into lower chambers were fixed with 5\% PFA and immunostained with crystal violet. The results of Transwell assay were subsequently quantified using a Leica inverted microscope fluorescent microscope (DM-IRB; Leica Microsystems GmbH, Wetzlar, Germany). The invasive capability was determined by counting the total number of invaded cells under each experimental condition and normalized to the number for the control condition.

In vivo implantation of tumors. The H510A cells were transduced with the lentiviruses for $8 \mathrm{~h}$ and then cultured for an additional $24 \mathrm{~h}$. The cells were then re-suspended and $10^{6}$ cells were implanted subcutaneously into the rear flanks of nude mice. The nude mice were obtained from Model Animal Research Center (Nanjing, China). In total, 12 mice (age, 4-6 weeks; average weight, $16.1 \mathrm{~g}$ ) were used in this study (i.e., 6 in the Lenti-C and 6 in the Lenti-450 group). The animal experiments and procedures were approved and reviewed by the Ethics Committee of Shanghai General Hospital, Shanghai Jiaotong University School of Medicine. The in vivo volume of tumors was determined weekly (length $\mathrm{x}$ width $\mathrm{x}$ height). After 6 weeks, all mice were sacrificed by an overdose of sodium amobarbital for Ki-67 immunostaining (Sigma).

Dual-luciferase reporter assay. To identify potential targets of miR-450, we utilized several miRNAtargets prediction tools, such as TargetScan (www.targetscan.org) PicTar (pictar.mdcberlin.de) and miRDB (www.mirdb.org). The IRF2 gene was amplified from a human lung cDNA library and verified. The 3'-UTR of IRF2 with predicted binding sites to hsa-miR-450 was cloned into the Xbal immediately downstream of Renilla 
luciferase reporter plasmid phRL-TK (Promega, Madison, WI, USA) leading to the wild-type IRF2 luciferase reporter plasmids (IRF2 3' UTR WT). The binding site of hsa-miR-450 on the IRF2 3'-UTR was also mutated by a Quik-Change ${ }^{\mathrm{TM}}$ Site-Directed Mutagenesis kit (Stratagene, La Jolla, CA, USA). The mutated IRF2 3'-UTR was then incorporated into phRL-TK to create the mutated IRF2 luciferase reporter plasmid (IRF2 3' UTR MUT). In 293T cells (obtained from the Shanghai Institute of Cell Biology), transfection with Lenti-miR-450 with IRF2 3' UTR (WT), IRF2 3' UTR (MUT) or an empty Renilla luciferase reporter plasmid (control) was performed for $36 \mathrm{~h}$. The relative luciferase units (RLU) were then measured by a dual-luciferase reporter assay (Promega) following the manufacturer's instructions.

Western blot analysis. The H510A and H2291 cells were harvested with cell lysis buffer containing $15 \%$ glycerol and 2\% (Sigma). The protein extracts (100 $\mu \mathrm{g}$ each) were dissolved by $10 \%$ SDS-PAGE and transferred onto nitrocellulose membranes (Bio-Rad, Hercules, CA, USA). The membranes were then incubated with antibodies against human IRF2 (1:500; Santa Cruz Biotechnology, Santa Cruz, CA, USA) at $4^{\circ} \mathrm{C}$ overnight, and HRP-conjugated secondary antibodies $(1: 103)$ at $20^{\circ} \mathrm{C}$ for $1.5 \mathrm{~h}$. $\beta$-actin was used as an internal control. The blots were visualized with an enhanced chemiluminescence film system (Amersham Pharmacia Biotech, Shanghai, China).

IRF2 overexpression assay. Whole sequences of IRF2 were cloned into a recombinant plasmid eukaryotic expression plasmid pcDNA3.1 (Sigma) to create the IRF2 overexpression plasmid, pcDNA3.1/IRF2, following the manufacturer's instructions. The transfection of pcDNA3.1/IRF2 and an empty pcDNA3.1 plasmid pcDNA3.1/(+) into H510A and H2291 cells were performed with Lipofectamine 2000 (Invitrogen). Twentyfour hours after transfection, the cells were re-suspended and plated in 96-well plates.

Statistical analysis. All experiments were performed at least in triplicate. The results are presented as the mean \pm standard error. Survival was evaluated with Kaplan-Meier curves and compared using a log-rank test. Statistical differences were measured using a Student's t-test using SPSS 16.0 software (SPSS Inc., Chicago, IL, USA) and a value of $\mathrm{P}<0.05$ was considered to indicate a statistically significant difference.

\section{Results}

miR-450 expression is decreased in lung cancer cells and correlates with survival. In the present study, we first used RT-qPCR to demonstrate the downregulation of miR-450 in established lung cancer cell lines in vitro, as well as in human samples in vivo. The results revealed that miR-450 was downregulated in the 7 lung cancer cell lines examined, H510A, H1703, SPC-A1, H522, H1299, SK-MES-1, H920 and H2291 compared with the normal human bronchial epithelial cell line (NuLi-1) (Fig. 1A; P<0.01). We also demonstrated that miR-450 expression was significantly decreased in the tumor $(\mathrm{T})$ tissues than in the corresponding non-tumor (ANT) tissues in the 11 patients with lung cancer (Fig. 1B; $\mathrm{P}<0.01$ ). In addition, we also found that the intrinsic expression of miR-450 exhibited
A
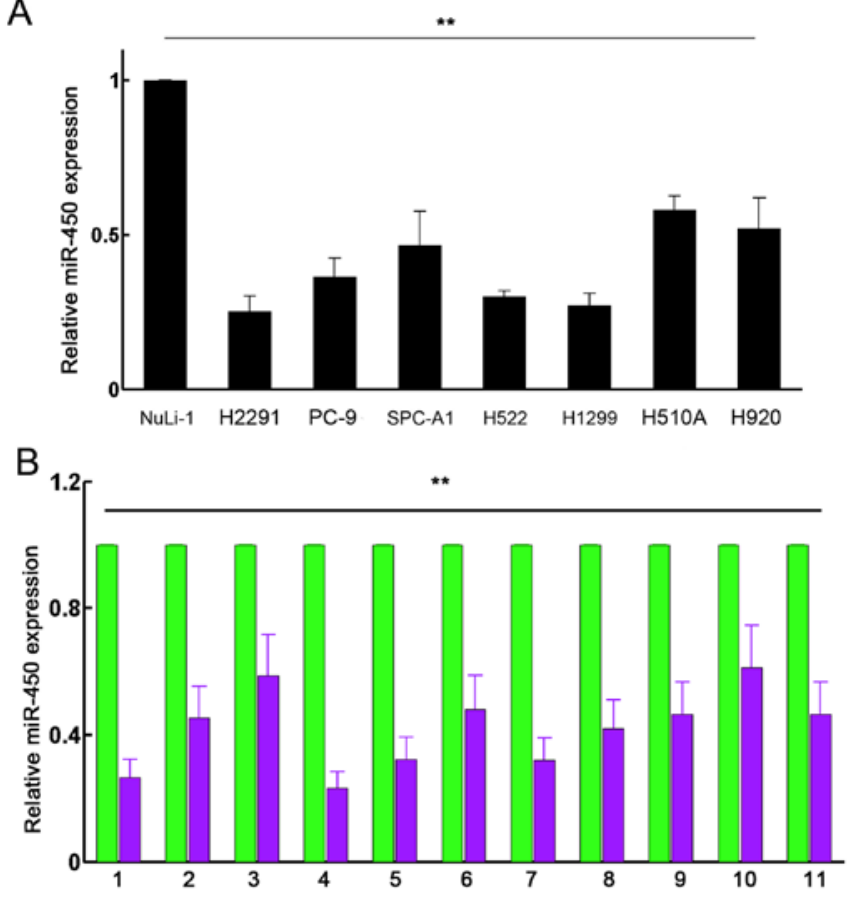

C

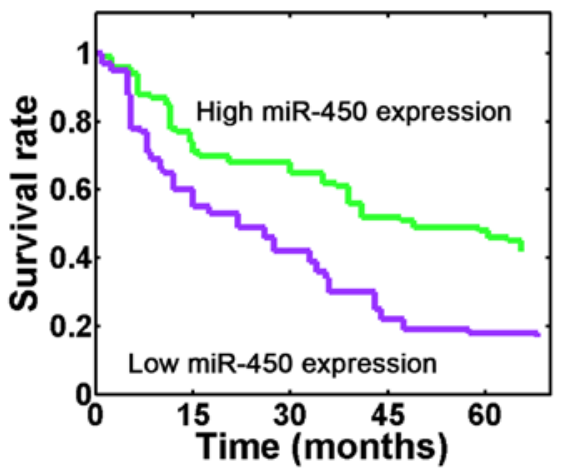

Figure 1. miR-450 expression is decreased in lung cancer. The results of qPCR demonstrated that miR-450 was decreased in lung cancer cells. (A) RT-qPCR was performed to examine the expression of miR-450 in 7 lung cancer cell lines, SPC-A1, H1703, H510A, H1299, H522, H920 and H2291 cells compared to that in a normal human bronchial epithelial cell line (NuLi-1); ${ }^{* *} \mathrm{P}<0.01$. (B) In samples from 11 patients with lung cancer, we used RT-qPCR to compare the expression of miR-450 between paired tumor ( $\mathrm{T}$; purple bars) tissues and corresponding adjacent non-tumor (ANT; green bars) tissues; ${ }^{* *} \mathrm{P}<0.01$. A pair-wise t-test was used. (C) Survival curves for low and high miR-450 expression. A log-rank test was performed. $\mathrm{P}=0.004$.

a significant correlation with the survival rates of the patients with lung cancer $(\mathrm{P}=0.004$; Fig. $1 \mathrm{C})$. These results suggest that miR-450 is frequently downregulated in lung cancer and that its downregulation correlates with poor survival.

Upregulation of miR-450 inhibits lung cancer cell invasion and proliferation. We transfected the H510A and H2291 cells with lentiviruses in order to increase the expression of miR-450. After $24 \mathrm{~h}$, the transfection efficiency was verified by RT-qPCR. The results revealed that the expression level of miR-450 was significantly increased by transfection with lentivirus containing miR-450 mimics (Lenti-miR-450) in both the H510A and H2291 cells compared with the control (Lenti-C) (Fig. 2A; $\mathrm{P}<0.05$ ). 
A
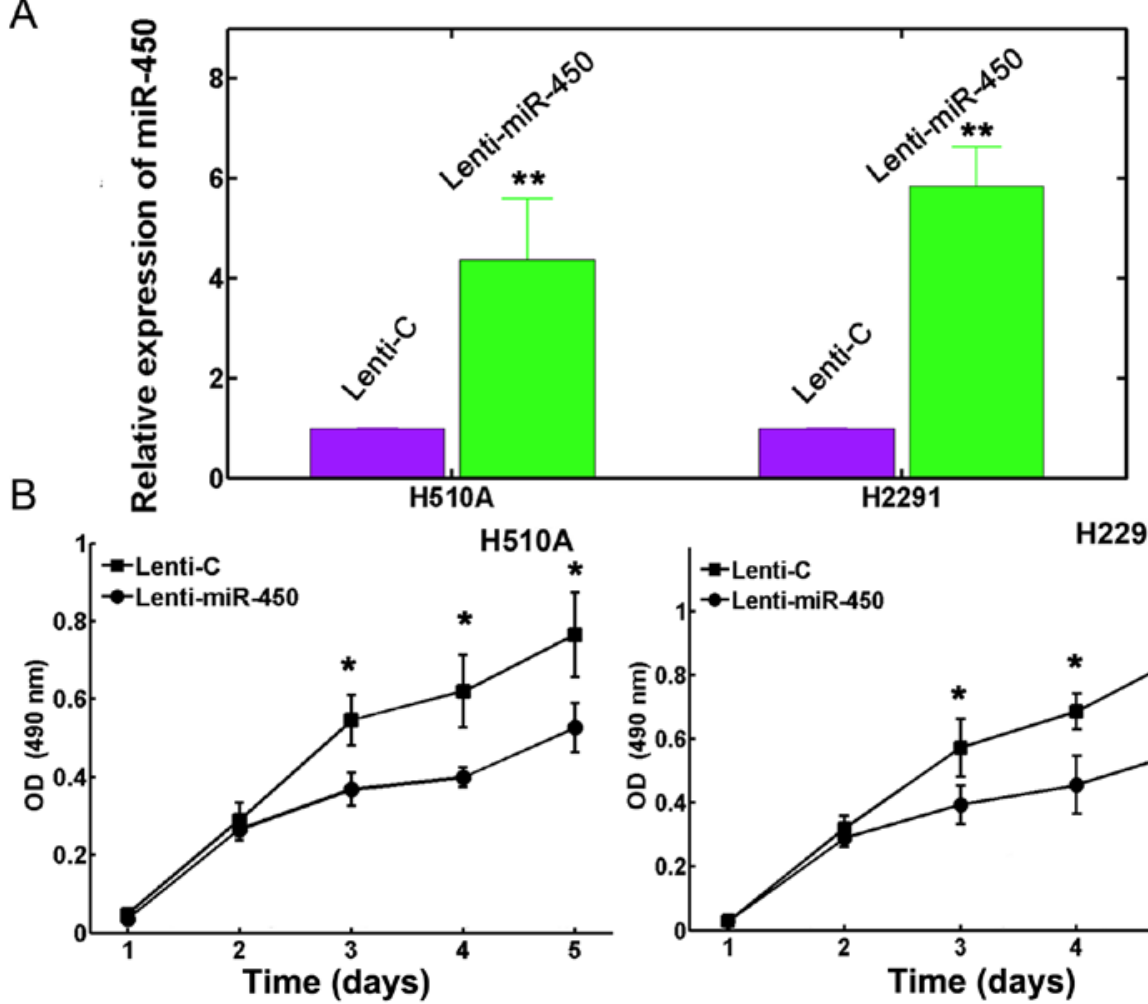

C
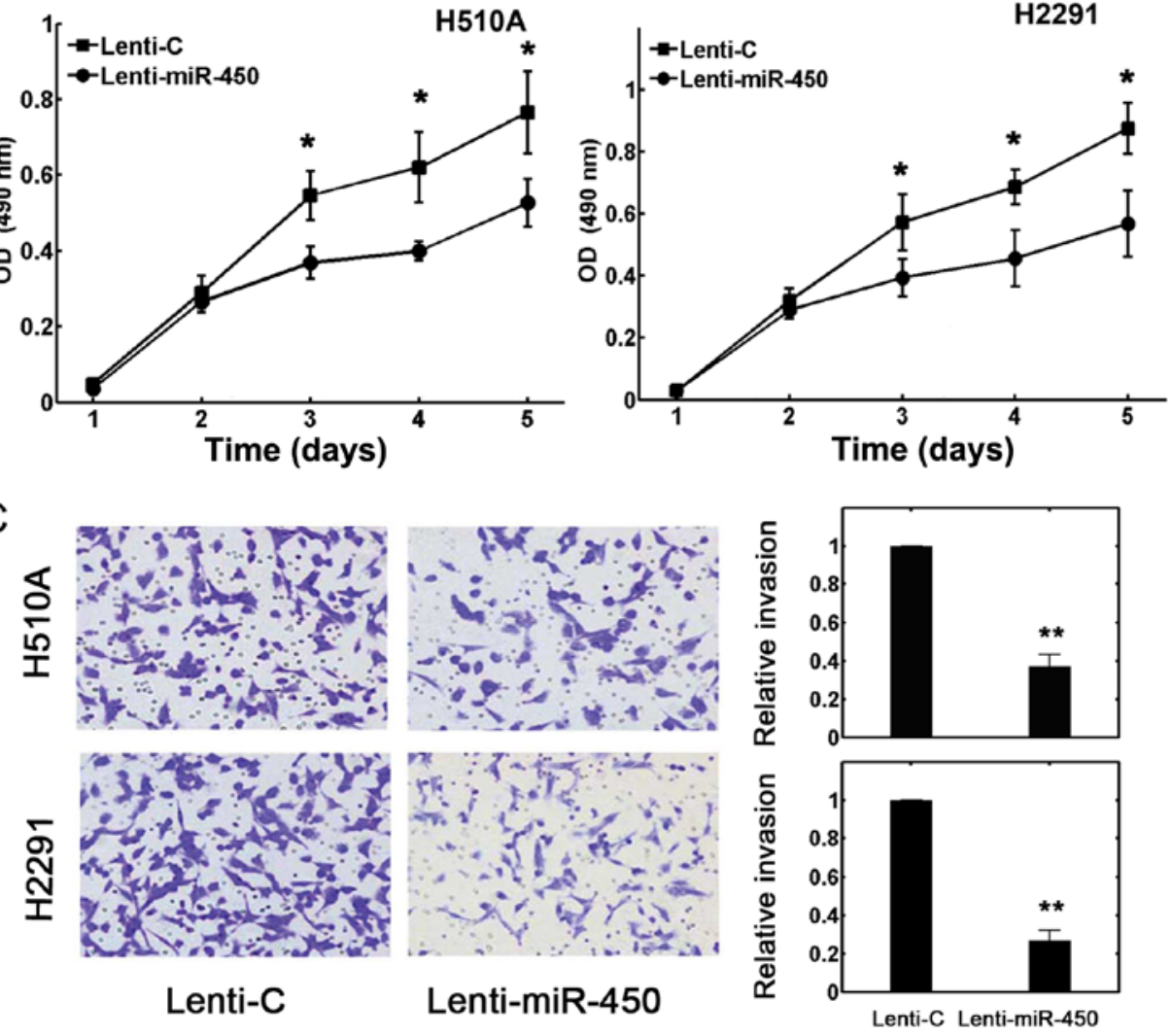

Figure 2. Evaluation of miR-450 transfection efficiency. (A) H510A and H2291 cells were transfected with lentivirus containing miR-450 mimics (Lenti-miR-450) or lentivirus linked with negative control miRNA (Lenti-C). After $24 \mathrm{~h}$, RT-qPCR was performed. (B) Lentivirus-transfected H510A and H2291 cells were subjected to MTT assay (5 days). (C) A Transwell assay to examine the invasion ability of H510A and H2291 cells. The invading cells into the lower chamber were stained with crystal violet (left panel). The invasive capabilities were also quantified (right panel). ${ }^{*} \mathrm{P}<0.05$ and ${ }^{* *} \mathrm{P}<0.01$ compared to control.

The H510A and H2291 cells transfected with lentivirus were re-suspended and plated in a 96-well plate for $120 \mathrm{~h}$. Cell proliferation assay was carried out every $24 \mathrm{~h}$ to determine the effects of miR-450 upregulation on lung cancer cell growth. The results revealed that the upregulation of miR-450 significantly attenuated lung cancer cell proliferation in both the H510A and H2291 cells (Fig. 2B; P<0.05). We also used a Transwell assay to examine the effects of miR-450 upregulation on lung cancer cell invasion. The results revealed that transfection with miR-450 mimics markedly reduced cell invasion (Fig. 2C, left panel). Quantification also confirmed that the upregulation of miR-450 reduced the invasive capabilities of the $\mathrm{H} 510 \mathrm{~A}$ and $\mathrm{H} 2291$ cells by $>50 \%$ (Fig. 2C, right panel; $\mathrm{P}<0.05$ ). Taken together, these data suggest that the upregulation of miR-450 inhibits the proliferation and invasion of lung cancer cells.
Upregulation of miR-450 inhibits the growth of implanted lung tumors in vivo. As we found that the upregulation of miR-450 inhibits lung cancer cell, we wished to determine whether miR-450 has a similar effect on lung tumor growth in vivo. Thus, we transfected the H510A cells with Lenti-miR-450 or Lenti-C for $24 \mathrm{~h}$. The cells were then re-suspended (the final cell number is $10^{6}$ ) and implanted subcutaneously into the rear flanks of nude mice. The sizes of the lung tumors, incuding length, width and height were measured each week and the total tumor volumes were determined. The results revealed that the growth capacity of the implanted lung tumors was significantly attenuated by the upregulation of miR-450 (Fig. 3A; P<0.05). Six weeks after implantation, the lung tumors were extracted and Ki-67 immunostaining was then performed. The results revealed 
A
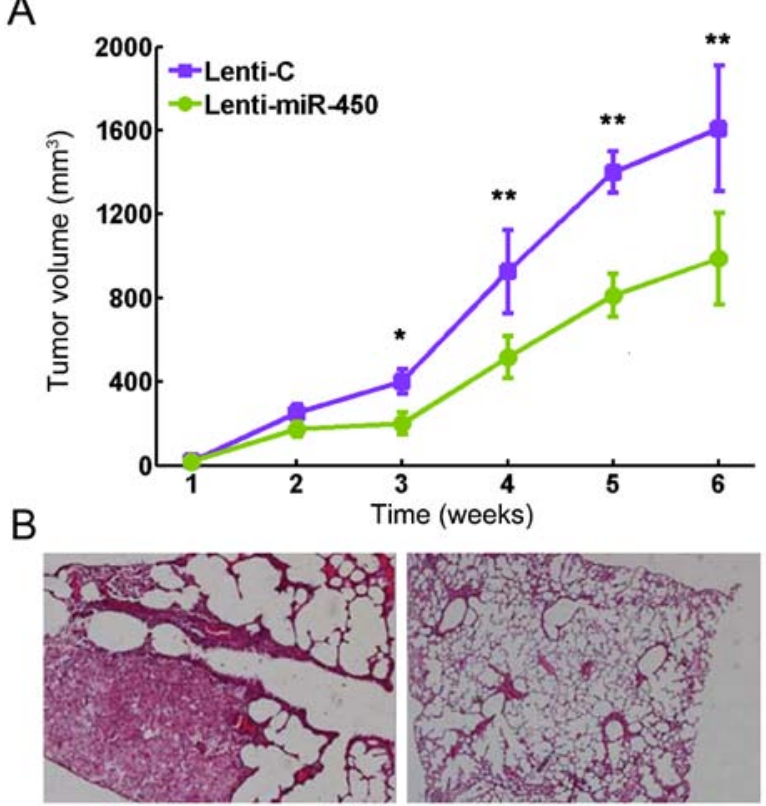

Lenti-C

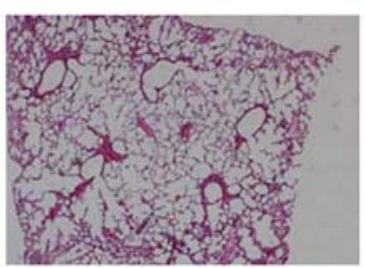

Lenti-miR-450

Figure 3. Upregulation of miR-450 attenuates the growth of implanted lung tumors. (A) Lentivirus-transfected H510A cells $\left(4 \times 10^{6}\right)$ were subcutaneously injected into the flanks of nude mice. The in vivo tumor volume was measured weekly (length $\mathrm{x}$ width $\mathrm{x}$ height) for 6 weeks. (B) By the end of the implantation, solid tumors were resected for $\mathrm{Ki}-67$ staining on paraffin-embedded sections. ${ }^{*} \mathrm{P}<0.05$ and ${ }^{* *} \mathrm{P}<0.01$ compared to control.

that Ki-67 staining was substantially decreased with the upregulation of miR-450 (Fig. 3B).

miR-450 directly targets IRF 2 in NSCLC. To identify potential targets of miR-450, we utilized several miRNAtargets prediction tools, such as TargetScan (www.targetscan.org) PicTar (pictar. mdc-berlin.de) and miRDB (www.mirdb.org) (Fig. 4A). The cross-verification of several databases indicated that that the oncogene, IRF2, may be a promising candidate (Fig. 4A and $\mathrm{B})$. We then performed dual-luciferase reporter assays using the $293 \mathrm{~T}$ cells. The results revealed that miR-450 can target luciferase plasmids with an intact or WT IRF2 3'-UTR and significantly reduced the RLU, whereas miR-450 had little effect on the luciferase plasmid containing a mutation (MUT) in the IRF2 3' UTR (Fig. 4C; P<0.05). We then paid more attention to two established NSCLC cell lines, H510A and H2291 cells, using western blot analysis and RT-qPCR to evaluate the profiles of IRF2 in the cells transfected with Lenti-miR-450. The results revealed that in both the H510A and H2291 cells, the protein level of IRF2 was markedly downregulated by the upregulation of miR-450 (Fig. 4D). The results of RT-qPCR confirmed this finding and showed that the expression of IRF2 in the H510A and H2291 cells was also downregulated by the upregulation of miR-450 (Fig. 4E; $\mathrm{P}<0.05$ ). Taken together, our data from dual-luciferase reporter assay, western blot analysis and RT-qPCR suggest that IRF2 may be the direct downstream target of miR-450 in both NSCLC and SCLC cells.

Upregulation of IRF 2 restores the malignant phenotypes of lung cancer cells following transfection with miR-450 mimics. We then conjectured that IRF2 may directly

A

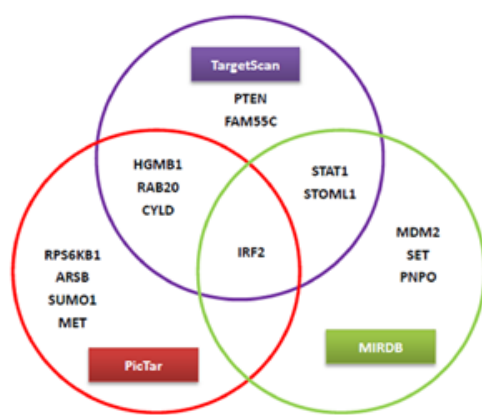

B

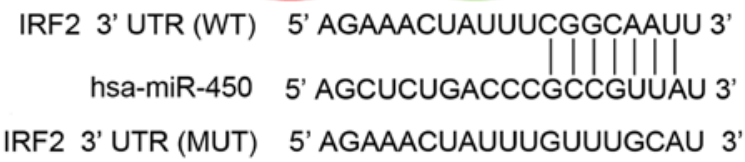

C

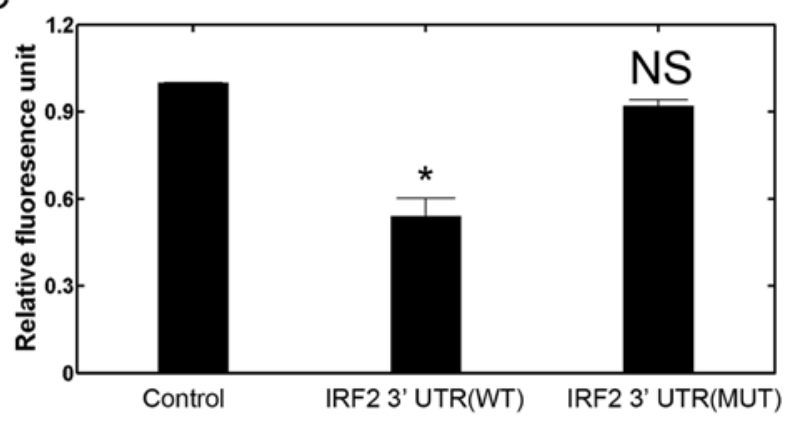

D

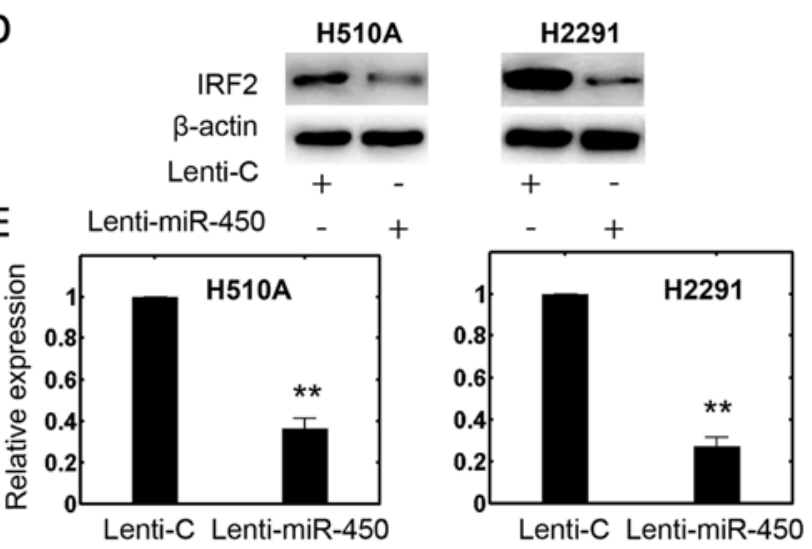

Figure 4. The interferon regulatory factor 2 (IRF2) is a direct target of miR-450. (A) Overlapping analysis demonstrated that IRF2 was identified as the sole target in 3 independent databases (PicTar, TargetScan and miRDB). (B) DNA sequences showed the alignment of hsa-miR-450 on wild-type (WT) IRF2 3'-UTR. The DNAs on the binding sites of IRF2 3'-UTR were also mutated (MUT). (C) In a dual-luciferase reporter assay, 293T cells were transfected with Lenti-miR-450, WT IRF2 plasmid (IRF2 3' UTR WT), mutated IRF2 plasmid with MUT miR-450 binding sties (IRF2-3'-UTR MUT) or an empty Renilla plasmid (control) for $24 \mathrm{~h}$. The luciferase activities of all constructs were quantified and normalized. (D) H510A and H2291 cells were transfected with lentivirus containing miR-450 mimics (Lenti-miR-450), or lentivirus containing negative control miRNA (Lenti-C) for $24 \mathrm{~h}$. The expression of IRF2 was examined by western blot analysis. (E) The mRNA expression of IRF2 was evaluated by RT-qPCR. ${ }^{*} \mathrm{P}<0.05$ and $^{* *} \mathrm{P}<0.01$ compared to control.

affect the miR-450-mediated inhibition of lung cancer cell proliferation and invasion. To verify this hypothesis, we constructed a mammalian plasmid to ectopically overexpress IRF2 (pcDNA3.1/IRF-2) in the H510A and H2291 cells. The efficiency of transfection was confirmed by RT-qPCR (Fig. 5A; $\mathrm{P}<0.05)$. We then performed a dual transfection assay. First, the H510A and H2291 cells were transfected with Lenti-miR-450. 


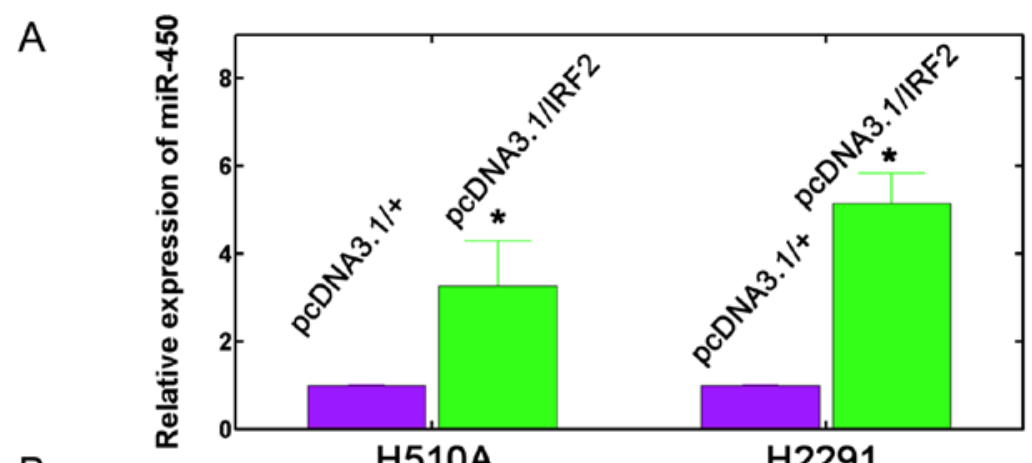

B
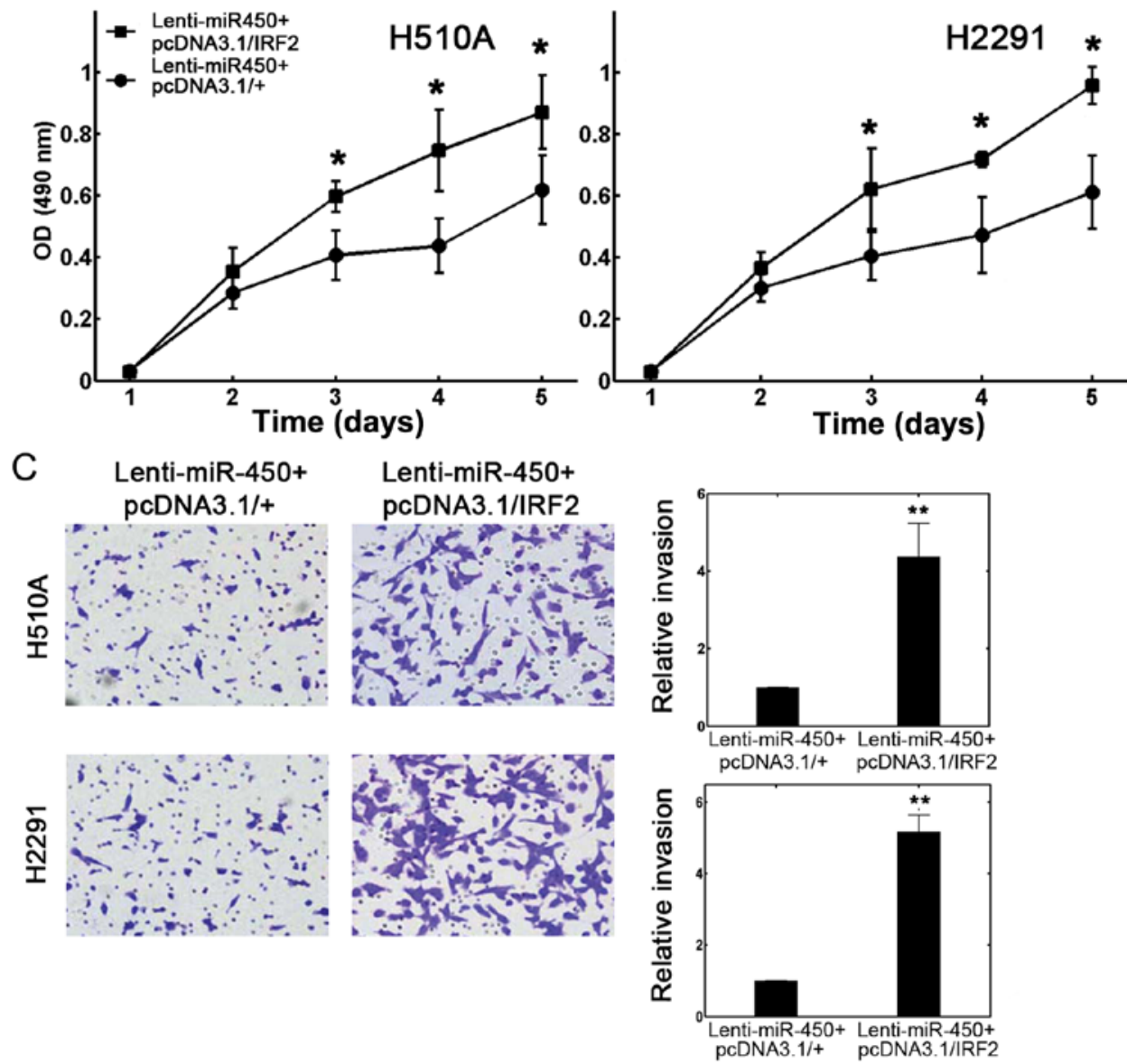

Figure 5. Overexpression of interferon regulatory factor 2 (IRF2) counteracts the inhibitory effects of miR-450 on lung cancer progression. (A) H510A and H2291 cells were transfected with IRF2 overexpression plasmid (pcDNA3.1/IRF2) or an empty plasmid pcDNA3.1/(+) for $24 \mathrm{~h}$. RT-qPCR was performed to examine the transfectino efficiency. (B) H510A and H2291 cells were transfected with Lenti-miR-450 for $24 \mathrm{~h}$ followed by co-transfection of pcDNA3.1/IRF2 or pcDNA3.1/(+) for a further $24 \mathrm{~h}$. Cell proliferation was then evaluated with a 5-day MTT assay. (C) Invasive capabilities of H510A and H2291 cells following co-transfection were quantified by Transwell assays. ${ }^{*} \mathrm{P}<0.05$ and ${ }^{* *} \mathrm{P}<0.01$ compared to control.

After $24 \mathrm{~h}$, the cells were transfected with eitherpcDNA3.1/IRF2 or the empty control plasmid pcDNA3.1/(+) for an additional $24 \mathrm{~h}$. A 5-day proliferation assay was then performed. The results revealed that IRF2 overexpression effectively restored lung cancer cell proliferation, which was initially attenuated by the upregulation of miR-450 in both the H510A and H2291 cells (Fig. 5B; P<0.05). Transwell assay also revealed that IRF2 overexpression significantly increased the number of invading cells into the lower chambers for both the H510A and H2291 cells (Fig. 5C, left panel). The invasive capability which was originally inhibited by miR-450 upregulation, ws elevated by $>200 \%$ with the overexpression of IRF2 (Fig. 5C, right panel; $\mathrm{P}<0.05)$. Therefore, all these results suggest that IRF2 directly mediates the regulatory effects of miR-450 on the proliferation and invasion of both NSCLC and SCLC cells.

\section{Discussion}

The miRNAs are small and non-coding RNAs, which serve as key regulators of gene expression by directly targeting mRNAs for translational repression. Deregulated miRNAs have been reported to be associated with the malignant progression of numerous types of cancer (22). These miRNAs may play regulatory and pivotal roles in the pathogenesis of 
tumors $(23,24)$. As previously reported, lung cancer patients usually suffer from tumor cell invasion and metastasis before diagnosis, rendering many present treatments such as surgery, radiotherapy and chemotherapy ineffective (25). Therefore, exploring the detailed mechanisms of lung cancer is critically important for development of novel therapeutics to improve overall survival rates.

Only a few studies to date have implied a role for miR-450. It has been demonstrated that miR-450a-3p regulates embryonic development by targeting Bub1 protein in mouse embryonic fibroblasts (MEFs) (26). Another study also implicated miR-450 in ER $\alpha$ signaling pathways (27). Furthermore, miR-450 can also be upregulated in TGF-9-treated PH5CH8 hepatocytes (28). However, a functional role of miR-450 in lung cancer has rarely been reported. In the present study, we first evaluated the pattern of miR-450 expression in several established lung cancer cell lines. We found that miR-450 was ubiquitously downregulated in all the tested lung cancer cell lines compared to normal bronchial cells. We also confirmed that miR-450 expression was significantly reduced in tumor tissues from lung cancer patients in comparison with normal adjacent tissues (Fig. 1). Moreover, transection with miR-450 mimics can stably increased miR-450 expression in the H510A or H2291 lung cancer cell lines (Fig. 2). Furthermore, the results of MTT and Transwell assays demonstrated that miR-450 suppresses the progression of lung cancer by inhibiting proliferation and invasion (Figs. 2 and 3). Therefore, from our preliminary experiments, it can be argued that miR-450 is a tumor suppressor, at least in NSCLC and SCLC cells.

The role of miRNAs in lung cancer development has been an active area of research. However, the majority of studies have mainly focused on either SCLC or NSCLC. In a previous study, Du et al argued that miR-337-3p can sensitize NSCLC cells to taxanes by targeting STAT3 and RAP1A, whereas the inhibition of miR-139-5p decreases SCLC cell viability through an unknown target (29). Furthermore, a recent miRNA profiling study aimed to distinguish NSCLC from SCLC by identifying unique miRNA expression patterns (30). However, few reports have investigated whether a single miRNA species can play a concordant role in both NSCLC and SCLC. In the current study, we found that miR-450 can mediate the tumor suppression of both SCLC and NSCLC cells by targeting IRF2. The overexpression of miR-450 inhibits the proliferation of both H510A and H2291 cells. Therefore, our study may instead unravel a novel facet of miRNA regulation in cancer development.

We further demonstrated that the transcription factor, IRF2, serves as a direct molecular target of miR-450 (Fig. 4). Upon miR-450 upregulation, not only lung cancer cell proliferation or invasion was inhibited, but IRF2 was also downregulated. That prompted us to further investigate whether IRF2 is directly involved in the regulation of miR-450 in lung cancer. Our IRF2 overexpression experiment partially confirmed this hypothesis, showing that the miR-450-induced inhibition on lung cancer cell proliferation and invasion was substantially restored by IRF2 overexpression. IRF1 and IRF2 have been shown to be upregulated by type I and type II interferons (31). In return, IFN- $\alpha$ and $-\beta$ can also be regulated by IRF family members (31). IRF1 has been reported to inhibit viral infection, particularly HCV (32). However, IRF2 was identified as a transcriptional repressor and can compete with the role of IRF1 (33). More importantly, IRF2 can also function as an oncogenic regulator. The deregulation of IRF2 has been reported in numerous tumor types. For example, Cui et al demonstrated that IRF2 was significantly upregulated in primary pancreatic cancer cells and correlated with a poor survival (34). Connett et al further argued that the level of IRF2 was maintained in tumor tissues and was associated with malignancy (35). In addition, it was also shown that IRF2 is involved in the negative feedback in the IFN- $\gamma$ pathway and has been implicated in tumor progression (36). In a more recent study, IRF2 was directly implicated in gastric cancer by modulating p53 expression (16). Therefore, miR-450 may target a universal oncogenic factor and exert its tumor suppressive role. Whether miR-450 is involved in other signaling pathways and plays undetermined roles remains to be clarified in future studies.

In conclusion, in this study, we established for the first time, to the best of our knowledge, a functional associatoin between miR-450 and human lung cancer. The upregulation of miR-450 expression exerts tumor suppressive effects on lung cancer. IRF2 was identified as the downstream target of miR-450 in lung cancer as it can counteract the effects of miR-450-induced tumor inhibition. The miR-450 and IRF2 signaling pathway should be further investigated in depth to uncover the epigenetic regulation of miRNAs in human lung cancer. This may help establish more elaborate targets in tumor intervention.

\section{References}

1. Siegel RL, Miller KD and Jemal A: Cancer statistics, 2015. CA Cancer J Clin 65: 5-29, 2015.

2. Siegel R, Naishadham D and Jemal A: Cancer statistics, 2012. CA Cancer J Clin 62: 10-29, 2012.

3. Xue C, Hu Z, Jiang W, Zhao Y, Xu F, Huang Y, Zhao H, Wu J, Zhang Y, Zhao L, et al: National survey of the medical treatment status for non-small cell lung cancer (NSCLC) in China. Lung Cancer 77: 371-375, 2012.

4. Miska EA: How microRNAs control cell division, differentiation and death. Curr Opin Genet Dev 15: 563-568, 2005.

5. Cai Y, Yu X, Hu S and Yu J: A brief review on the mechanisms of miRNA regulation. Genomics Proteomics Bioinformatics 7: 147-154, 2009 .

6. Feng B, Zhang K, Wang R and Chen L: Non-small-cell lung cancer and miRNAs: novel biomarkers and promising tools for treatment. Clin Sci (Lond) 128: 619-634, 2015.

7. Lewis BP, Burge CB and Bartel DP: Conserved seed pairing, often flanked by adenosines, indicates that thousands of human genes are microRNA targets. Cell 120: 15-20, 2005.

8. Kalia M: Biomarkers for personalized oncology: Recent advances and future challenges. Metabolism 64 (Suppl 1): S16-S21, 2015.

9. Zhang Y, Yang Q and Wang S: MicroRNAs: A new key in lung cancer. Cancer Chemother Pharmacol 74: 1105-1111, 2014.

10. Wang R, Wang ZX, Yang JS, Pan X, De W and Chen LB: MicroRNA-451 functions as a tumor suppressor in human non-small cell lung cancer by targeting ras-related protein 14 (RAB14). Oncogene 30: 2644-2658, 2011.

11. Liu X, Sempere LF, Ouyang H, Memoli VA, Andrew AS, Luo Y, Demidenko E, Korc M, Shi W, Preis M, et al: MicroRNA-31 functions as an oncogenic microRNA in mouse and human lung cancer cells by repressing specific tumor suppressors. J Clin Invest 120: 1298-1309, 2010.

12. Zhao Z, Liu J, Wang C, Wang Y, Jiang Y and Guo M: MicroRNA-25 regulates small cell lung cancer cell development and cell cycle through cyclin E2. Int J Clin Exp Pathol 7: 7726-7734, 2014.

13. Tanaka N, Toyooka S, Soh J, Kubo T, Yamamoto H, Maki Y, Muraoka T, Shien K, Furukawa M, Ueno T, et al: Frequent methylation and oncogenic role of microRNA-34b/c in small-cell lung cancer. Lung Cancer 76: 32-38, 2012.

14. Harada H, Taniguchi $\mathrm{T}$ and Tanaka $\mathrm{N}$ : The role of interferon regulatory factors in the interferon system and cell growth control. Biochimie 80: 641-650, 1998. 
15. Xi H, Eason DD, Ghosh D, Dovhey S, Wright KL and Blanck G: Co-occupancy of the interfearon regulatory element of the class II transactivator (CIITA) type IV promoter by interferon regulatory factors 1 and 2. Oncogene 18: 5889-5903, 1999.

16. Chen YJ, Wu H, Zhu JM, Li XD, Luo SW, Dong L, Liu TT, Shen XZ: MicroRNA-18a modulates P53 expression by targeting IRF2 in gastric cancer patients. J Gastroenterol Hepatol 31: 155-63, 2016.

17. Sakai T, Mashima H, Yamada Y, Goto T, Sato W, Dohmen T, Kamada K, Yoshioka M, Uchinami H, Yamamoto Y and Ohnishi $\mathrm{H}$ : The roles of interferon regulatory factors 1 and 2 in the progression of human pancreatic cancer. Pancreas 43: 909-916, 2014.

18. Choo A, Palladinetti P, Holmes T, Basu S, Shen S, Lock RB, O'Brien TA, Symonds G and Dolnikov A: siRNA targeting the IRF2 transcription factor inhibits leukaemic cell growth. Int J Oncol 33: 175-183, 2008.

19. Nicolini A, Carpi A and Rossi G: Cytokines in breast cancer Cytokine Growth Factor Rev 17: 325-337, 2006.

20. Wang Y, Liu DP, Chen PP, Koeffler HP, Tong XJ and Xie D: Involvement of IFN regulatory factor (IRF)-1 and IRF-2 in the formation and progression of human esophageal cancers. Cancer Res 67: 2535-2543, 2007.

21. Xi H and Blanck G: Interferon regulatory factor-2 point mutations in human pancreatic tumors. Int J Cancer 87: 803-808, 2000

22. Calin GA, Sevignani C, Dumitru CD, Hyslop T, Noch E, Yendamuri S, Shimizu M, Rattan S, Bullrich F, Negrini M and Croce CM: Human microRNA genes are frequently located at fragile sites and genomic regions involved in cancers. Proc Nat Acad Sci USA 101: 2999-3004, 2004.

23. Dykxhoorn DM: MicroRNAs and metastasis: Little RNAs go a long way. Cancer Res 70: 6401-6406, 2010.

24. Hummel R, Hussey DJ and Haier J: MicroRNAs: Predictors and modifiers of chemo- and radiotherapy in different tumour types. Eur J Cancer 46: 298-311, 2010.

25. Zhao Y, Wei Q, Hu L, Chen F, Hu Z, Heist RS, Su L, Amos CI, Shen $\mathrm{H}$ and Christiani DC: Polymorphisms in MicroRNAs are associated with survival in non-small cell lung cancer. Cancer Epidemiol Biomarkers Prev 23: 2503-2511, 2014

26. Luo M, Weng Y, Tang J, Hu M, Liu Q, Jiang F, Yang D, Liu C, Zhan X, Song P, et al: MicroRNA-450a-3p represses cell proliferation and regulates embryo development by regulating Bub1 expression in mouse. PLoS One 7: e47914, 2012.
27. Castellano L, Giamas G, Jacob J, Coombes RC, Lucchesi W, Thiruchelvam P, Barton G, Jiao LR, Wait R, Waxman J, et al: The estrogen receptor-alpha-induced microRNA signature regulates itself and its transcriptional response. Proc Natl Acad Sci USA 106: 15732-15737, 2009.

28. Brockhausen J, Tay SS, Grzelak CA, Bertolino P, Bowen DG, d'Avigdor WM, Teoh N, Pok S, Shackel N, Gamble JR, et al: miR-181a mediates TGF- $\beta$-induced hepatocyte EMT and is dysregulated in cirrhosis and hepatocellular cancer. Liver Int 35: 240-253, 2015

29. Du L and Pertsemlidis A: microRNA regulation of cell viability and drug sensitivity in lung cancer. Expert Opin Biol Ther 12: 1221-1239, 2012.

30. Du L, Schageman JJ, Irnov, Girard L, Hammond SM, Minna JD, Gazdar AF and Pertsemlidis A: MicroRNA expression distinguishes SCLC from NSCLC lung tumor cells and suggests a possible pathological relationship between SCLCs and NSCLCs. J Exp Clin Cancer Res 29: 75, 2010.

31. Masumi A, Ito M, Mochida K, Hamaguchi I, Mizukami T, Momose H, Kuramitsu M, Tsuruhara M, Takizawa K, Kato A and Yamaguchi K: Enhanced RIG-I expression is mediated by interferon regulatory factor- 2 in peripheral blood $\mathrm{B}$ cells from hepatitis $\mathrm{C}$ virus-infected patients. Biochem Biophys Res Commun 391: 1623-1628, 2010.

32. Ciccaglione AR, Stellacci E, Marcantonio C, Muto V, Equestre M, Marsili G, Rapicetta M and Battistini A: Repression of interferon regulatory factor 1 by hepatitis $C$ virus core protein results in inhibition of antiviral and immunomodulatory genes. J Virol 81: 202-214, 2007.

33. Ikushima H, Negishi $H$ and Taniguchi T: The IRF family transcription factors at the interface of innate and adaptive immune responses. Cold Spring Harb Symp Quant Biol 78: 105-116, 2013.

34. Cui L, Deng Y, Rong Y, Lou W, Mao Z, Feng Y, Xie D and Jin D: IRF-2 is over-expressed in pancreatic cancer and promotes the growth of pancreatic cancer cells. Tumour Biol 33: 247-255, 2012.

35. Connett JM, Badri L, Giordano TJ, Connett WC and Doherty GM: Interferon regulatory factor 1 (IRF-1) and IRF-2 expression in breast cancer tissue microarrays. J Interferon Cytokine Res 25: 587-594, 2005.

36. Wang Y, Liu D, Chen P, Koeffler HP, Tong X and Xie D: Negative feedback regulation of IFN-gamma pathway by IFN regulatory factor 2 in esophageal cancers. Cancer Res 68: 1136-1143, 2008. 